\title{
Modeling diurnal variations of the IAR parameters
}

\author{
E. N. Fedorov' ${ }^{1}$ N. G. Mazur ${ }^{1}$ - V. A. Pilipenko ${ }^{2}$. \\ E. N. Ermakova $^{3}$
}

Received: 10 August 2015/Accepted: 23 December 2015/Published online: 12 January 2016

(C) Akadémiai Kiadó 2016

\begin{abstract}
Characteristic feature of the upper ionosphere is the occurrence of the ionospheric Alfvén resonator (IAR) and MHD waveguide, which can trap the electromagnetic waves in the frequency range from fractions of $\mathrm{Hz}$ to few $\mathrm{Hz}$. The proposed numerical model is based on the solution of MHD equations in a realistic ionosphere, whose parameters are reconstructed from the IRI model. We estimated, both analytically and numerically, a critical, wave scale dependent, value of the Hall conductance when a wave equation for an uncoupled Alfvénic mode can be used to estimate the spectral parameters of the IAR. The model has enabled us to compare the contributions into the IAR Q-factor of the Joule dissipation in the lower ionosphere and a wave leakage into the magnetosphere. The first mechanism dominates during daytime, whereas the latter mechanism prevails during nighttime. The ground signatures of IAR can be used for monitoring of the F-layer plasma density and vertical total electron content on the basis of relationships derived from the developed IAR model.
\end{abstract}

Keywords ULF waves · Geomagnetic pulsations · Ionospheric Alfvén resonator · Mode coupling · Ionosphere monitoring

\section{Introduction: MHD resonators and waveguides in the upper ionosphere}

The natural MHD resonators and waveguides play an important role in space physics. Their occurrence leads to the possibility of a significant wave energy accumulation in certain regions of the outer space, where this wave power can influence the particle

V. A. Pilipenko

space.soliton@gmail.com

Institute of Physics of the Earth, Moscow, Russia

2 Space Research Institute, Moscow, Russia

3 Radiophysics Research Institute, N. Novgorod, Russia 
dynamics. A MHD resonator can form a narrow-band multi-spectral structure of ultra-lowfrequency (ULF) emissions, which can be used as a tool of "hydromagnetic spectroscopy" of the outer space environment.

Since theoretical prediction of the existence of the Alfvén quasi-resonances in the ionosphere (Polyakov 1976; Polyakov and Rapoport 1981) the Ionospheric Alfvén Resonator (IAR) was intensively studied. IAR is formed due to a partial reflection of Alfvén waves from a steep gradient of the vertical profile of Alfvén velocity $V_{A}(z)$ in the upper ionosphere. The IAR signatures were found at mid-latitude (Belyaev et al. 1987, Belyaev et al. 1990; Kulak et al. 1999; Molchanov et al. 2004) and low-latitude (Bösinger et al. 2004) stations, at auroral latitudes (Belyaev et al. 1999), and even poleward from the auroral oval (Semenova and Yahnin 2008). More complete references can be found in reviews by Demekhov (2012) and Pilipenko (2012). At the ground the IAR multiband spectral signatures are observed nearly every night, but never during daytime. In the auroral zone (Yahnin et al. 2003) the IAR observation probability also increases at night, but it is lower than at low latitudes. The enhanced geomagnetic activity hinders the midlatitude IAR observations (Parent et al. 2010). The IAR signatures on the ground at midlatitude are regularly observed in years of minimum solar activity, and are practically absent in years of maximum solar activity (Belyaev et al. 2000).

The idea of Alfvén resonator also became popular in the solar (Leroy 1980) and planetary physics. The IAR formation along an inhomogeneous Jupiter-Io flux tube was suggested to explain periodic modulation of Jovian electromagnetic emission ( $\mathrm{S}$ bursts) (Ergun et al. 2006).

The IAR in the terrestrial ionosphere can be excited by various mechanisms, comprising magnetospheric, ionospheric, atmospheric sources, and active experiments. At auroral latitudes the IAR can be driven by a magnetospheric source, e.g. non-steady field-aligned current, and even may become unstable due to the feedback instability in the coupled magnetosphere-ionosphere system (Trakhtengertz and Feldstein 1991; Lysak 1991; Pokhotelov et al. 2001). Small-scale kinetic Alfvén waves trapped in the IAR at auroral latitudes are supposedly responsible for the electron acceleration causing activation of auroral forms (Chaston et al. 2002). The IAR influence was suggested as a possible mechanism which determines a central frequency of auroral Pi1c emission (Woodroffe and Lysak 2012). This suggestion is in accord with the increase of the auroral Pi1 emission mid-frequency from $\sim 0.2 \mathrm{~Hz}$ during daytime to $\sim 0.3-1.0 \mathrm{~Hz}$ during midnight (Heacock 1967), similar to IAR eigenfrequency diurnal variation.

At middle and low latitudes a possible energy source for the IAR excitation is related to the distant global lightning centers in tropics (Belyaev et al. 1989). Additionally, regional and near-by thunderstorms are able to generate signals in the IAR range (Fedorov et al. 2006; Surkov et al. 2006; Schekotov et al. 2011).

The possibility of the IAR excitation by the fluctuations of neutral gas velocity at the heights of the ionospheric E-layer was theoretically predicted by Molchanov et al. (2004), and Surkov et al. (2004). Fluctuations of the neutral particle velocity result in the alternating ionospheric current, which radiates MHD waves into the upper ionosphere. However, this possibility has not been observationally validated yet.

Alfvénic pulse in the ionosphere and IAR response can be generated by an active manmade impact on the ionosphere, e.g. chemical release experiment (Gaidukov et al. 1993), rocket exhaust gases (Deminov et al. 2001), or modulated radioheating of the ionosphere (Trakhtengerts et al. 2000; Pashin et al. 2003).

Different excitation mechanisms result in different waveforms of the IAR response upon an external driving. The multi-band spectral structure can be produced either by a 
superposition of oscillatory transients or a sequence of pulses with a regular time delay (Fedorov et al. 2014).

The frequency difference between adjacent harmonics $\Delta f$ was suggested to be used as an effective tool for monitoring of the upper ionosphere density with ground magnetometers (Potapov et al. 2014). The usage of IAR signatures for the spectroscopy of the top-side ionosphere requires a numerical model based on a realistic profile of the plasma constituents. Any approach to the interpretation of ionosphere resonance features demands a knowledge of spectral and dissipative properties of the IAR. Moreover, the sensitivity of modern electric field sensors and magnetometers onboard low-orbiting satellites became high enough to detect the IAR signatures in the top-side ionosphere (Simões et al. 2012; Dudkin et al. 2014). To interpret correctly the satellite observations an adequate model of the IAR eigenmode altitude structure is necessary.

For the interpretation of basic IAR features mostly the theoretical models where the refractive index $n_{A}(z)$ in the upper ionosphere was approximated by some functions, enabling an analytical solution, were applied (Polyakov and Rapoport 1981; Lysak 1993; Demekhov et al. 2000; Hebden et al. 2005). In this approach, the bottom ionosphere was modeled by a thin layer with the height-integrated Pedersen conductance $\Sigma_{P}$. The coupling of Alfvén and fast magnetosonic modes was neglected. However, such models could not adequately account for the realistic plasma profile in the upper ionosphere and dissipative properties of the lower ionosphere. Therefore, in more recent IAR models (Ermakova et al. 2000, 2008; Bösinger et al. 2009; Lysak et al. 2013), realistic profile stemming from the International Reference Ionosphere (IRI) program and inclined geomagnetic field have been used (Bilitza and Reinisch 2008).

The model proposed in this paper is more adequate as compared with most earlier studies, and is based on the realistic ionosphere modeled by the IRI model. The proposed model is aimed to describe quantitatively not only the spectral, but dissipative as well, parameters of the IAR. The results of this model have been calculated for the conditions corresponding to the data from mid-latitude station Moshiri (geomagnetic latitude $35.6^{\circ}$ ).

\section{Model of MHD waves in the ionosphere}

We introduce a model of the magnetosphere - ionosphere - atmosphere - ground system which will be used to describe the properties of MHD waves in an inhomogeneous ionosphere. We use the Cartesian coordinate system, where the $x, y$ and $z$-axes are directed southward, eastward, and upward, respectively; $z=0$ at the Earth surface. The magnetic field inclination (dip angle) is denoted as $I(I>0$ in the Northern and $I<0$ in the Southern hemisphere; vertical $\mathbf{B}_{0}$ corresponds to $I= \pm \pi / 2$ ). We also introduce the non-rectangular coordinate system $\left\{x^{1}, x^{2}, x^{3}\right\}$ with horizontal coordinate planes $x^{3}=$ const (Fig. 1). This coordinate system is related to the Cartesian coordinate system with relationships $x=x^{1}+x^{3} \cot I, y=x^{2}$, and $z=x^{3}$. In this system, coordinate lines $x^{3}$ coincide with magnetic field lines; the distance from the Earth surface along a field line is $s=x^{3} / \sin I$.

For low frequencies (Pc3-5 and Pi2 pulsations) the approximation of the thin layer ionosphere may be used. The most complete analytical relationships of the general theory of MHD wave interactions with a thin ionosphere can be found in (Alperovich and Fedorov 2007). However, this theory cannot be used for waves in the IAR frequency range, whose field-aligned wave length is comparable to the scale of ionospheric inhomogeneity. 
Fig. 1 A sketch of the coordinate system on a plane $y=$ $x^{2}=$ const. The unit vectors of the Cartesian $\{x, y, z\}$ and nonorthogonal $\left\{x^{1}, x^{2}, x^{3}\right\}$ are shown. Dashed lines denote a projection onto coordinate lines of the non-orthogonal system. The relationship between the non-orthogonal and Cartesian coordinates is given in the text

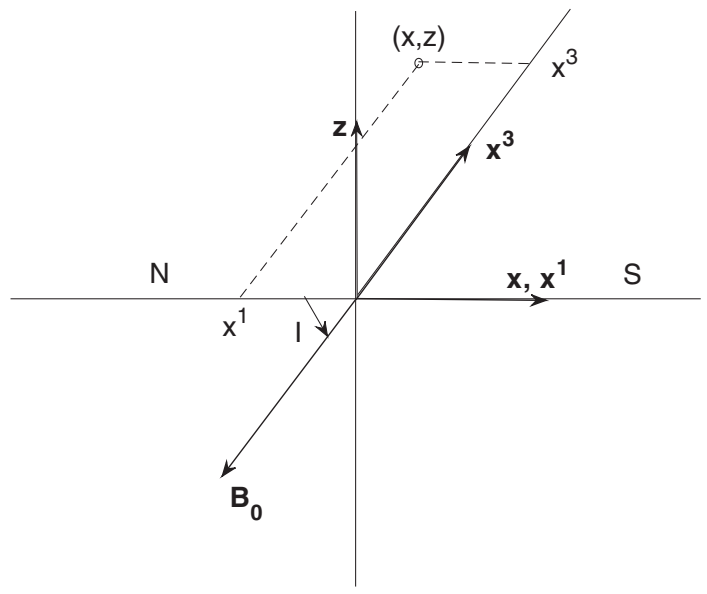

Therefore, a complete set of coupled wave equations in an inhomogeneous medium must be treated.

The atmosphere and the ground are assumed to be isotropic conductors with conductivities $\sigma_{a}$ and $\sigma_{g}$. The system parameters do not vary in the horizontal direction, i.e. along $x^{1}$ and $x^{2}$. Magnetospheric and ionospheric plasma are characterized by the tensor of complex dielectric permittivity $\hat{\varepsilon}(z, \omega)$.

The wave electric (e) and magnetic (b) fields are decomposed into two modes. The magnetospheric wave fields are the sum of an Alfvén mode (where the disturbed magnetic field $\mathbf{b}_{\perp}$ is perpendicular to $\mathbf{B}_{0}$ and divergence-free, $\nabla \cdot \mathbf{b}_{\perp}=0$, whereas the longitudinal component is vanishing $b_{\|}=0$ ); and a fast magnetosonic (FMS) mode (where $\mathbf{b}_{\perp}$ is curlfree, $\nabla \times \mathbf{b}_{\perp}=0$, whereas the field-aligned component of the current vanishes $j_{\|}=0$ ). In its turn, an electromagnetic disturbance in the atmosphere and ground is composed of a electric (TH) E-mode and magnetic (TE) H-mode.

The wave structure across $B_{0}$ is characterized by the wave vector components in the N-S direction $k_{1}$ and E-W direction $k_{2}$, as follows $\propto \exp \left(-i \omega t+i k_{1} x_{1}+i k_{2} x_{2}\right)$. The waves are assumed to be elongated in the azimuthal direction, so $k_{2}=0$ and $k=k_{1}$. In this case, the Alfvén mode has an azimuthal magnetic component $b_{2}$ only, whereas the wave electric field with component $e_{1}$ lies in the meridional plane and is transverse to $\mathbf{B}_{0}$. The FMS mode has non-vanishing azimuthal electric component $e_{2}$, radial magnetic component $b_{1}$, and compressional component $b_{3} \equiv b_{\|}$, whereas the azimuthal component $b_{2}=0$.

Owing to a high resistance of the atmosphere, the current density near the Earth's surface is much less than the current density of ionospheric currents. Therefore, the ratio of magnetic components of the atmospheric E-mode to the magnetic components in the ionosphere is very small. In other words, the E-mode is very weakly excited by magnetospheric disturbances, and its contribution into the wave field will not be considered further.

The wave structure is determined from the solution of Maxwell's equations with the complex permittivity tensor $\hat{\varepsilon}$, which in a coordinate system with $\mathbf{B}_{0}$-aligned $z$-axis looks as follows 


$$
\hat{\varepsilon}=\left(\begin{array}{ccc}
\varepsilon_{\perp} & i g & 0 \\
-i g & \varepsilon_{\perp} & 0 \\
0 & 0 & \varepsilon_{\|}
\end{array}\right) .
$$

The tensor components $\varepsilon_{\perp}$ and ig are calculated below numerically using the cold collisional plasma approximation (Ginzburg 1970). In an oblique-angled coordinate system under the assumption $\varepsilon_{\|} \rightarrow \infty$, Maxwell's equations are reduced to the following system

$$
\begin{aligned}
& \partial_{3} b_{1}=i k(\cot I) b_{1}-\omega \mu_{0} g(\sin I)^{-1} e_{1}+i\left(k^{2} / \omega-\omega \mu_{0} \varepsilon_{\perp}\right) e_{2}, \\
& \partial_{3} b_{2}=i \omega \mu_{0} \varepsilon_{\perp}(\sin I)^{-2} e_{1}-\omega \mu_{0} g(\sin I)^{-1} e_{2}, \\
& \partial_{3} e_{1}=i \omega b_{2} \\
& \partial_{3} e_{2}=-i \omega b_{1}+i k(\cot I) e_{2} .
\end{aligned}
$$

Here $\mu_{0}$ and $\varepsilon_{0}$ are the vacuum permeability and permittivity, respectively. If the wave frequency $\omega$ is much less than any ion gyrofrequency $\Omega$, then in the E-layer $\varepsilon_{\perp} \simeq i \sigma_{P} / \omega$, $g=\sigma_{H} / \omega$, and in the F-layer $\varepsilon_{\perp} \simeq \varepsilon_{0} c^{2} / V_{A}^{2}$, whereas off-diagonal terms are small, $g \approx 0$. A model with a full ionosphere and tilted field lines was also developed by Sciffer et al. (2005) using essentially the same equations, although they did not focus on the IAR.

If the Hall conductivity $\sigma_{H}$ can be neglected, then the basic system (2) splits into two uncoupled sub-systems for Alfvén waves

$$
\begin{gathered}
\partial_{3} e_{1}=i \omega b_{2}, \\
\partial_{3} b_{2}=i \omega \mu_{0} \varepsilon_{\perp}(\sin I)^{-2} e_{1},
\end{gathered}
$$

and for FMS waves

$$
\begin{gathered}
\partial_{3} e_{2}=-i \omega b_{1}+i k(\cot I) e_{2}, \\
\partial_{3} b_{1}=i k(\cot I) b_{1}+i\left(k^{2} / \omega-\omega \mu_{0} \varepsilon_{\perp}\right) e_{2} .
\end{gathered}
$$

Limitations on a possible usage of reduced system (3) for the estimation of IAR spectral properties will be discussed in Sect. 6.

\section{Numerical model of the IAR}

To reconstruct the altitude profile of the complex permittivity tensor (1) for the basic Eq. (2) the IRI2007 program has been used. It is an empirical model computing height profiles of electron density $N_{e}$, ion composition $\left(\mathrm{O}^{+}, \mathrm{N}^{+}, \mathrm{H}^{+}, \mathrm{He}^{+}, \mathrm{O}_{2}^{+}, \mathrm{NO}^{+}\right)$, ion density $N_{i}$, electron $T_{e}$ and ion $T_{i}$ temperatures, in the altitude range from $60 \mathrm{~km}$ up to the ionosphere-magnetosphere interface $H=2000 \mathrm{~km}$ with cadence $1 \mathrm{~km}$ for a specified date and location. This model incorporates the COSPAR International Reference Atmosphere 1986 (CIRA-86) neutral temperature model and International Geomagnetic Reference Field (IGRF) model. In addition the model MSIS has been used to obtain temperature $T_{n}$ and concentration of neutral particles ( $\mathrm{He}, \mathrm{O}, \mathrm{N}_{2}, \mathrm{O}_{2}, \mathrm{Ar}, \mathrm{H}, \mathrm{N}$ ).

Using these parameters the altitude profiles of the electron and ion collision frequencies $v_{e}$ and $v_{i}$, and the tensor components $\varepsilon_{\perp}(z, \omega)$ and $i g(z, \omega)(1)$, have been calculated using general formulas from (Ginzburg 1970). Also the important ionospheric parameters determined by the tensor (1), such as the complex plasma conductivities $\sigma_{1}(z, \omega)=-i \omega \varepsilon_{\perp}(z, \omega), \quad \sigma_{2}(z, \omega)=\omega g(z, \omega), \quad \sigma_{3}(z, \omega)=-i \omega \varepsilon_{\|}(z, \omega)$, and refraction 
index $n(z, \omega)$ have been calculated. The frequency dependence of $\sigma_{1}(z, \omega)$ and $\sigma_{2}(z, \omega)$ become negligible for low frequencies $\omega / \Omega \ll 1$. In this case complex conductivities reduce to static Pedersen and Hall conductivities, $\sigma_{1}(z, \omega) \simeq \sigma_{P}$ and $\sigma_{2}(z, \omega) \simeq \sigma_{H}$. Above the E-layer the refraction index of low frequency waves is $n_{A}(z, \omega) \simeq c$ / $V_{A}(z)=\operatorname{Re} \sqrt{\varepsilon_{\perp} / \varepsilon_{0}}$. These approximate relationships can be used for waves in the ionosphere with frequencies up to several Hz. Besides finite-frequency effects $\propto \omega / \Omega$, we neglect the coupling between Alfvén and FMS modes due to nonzero Hall conductivity. A validity of this assumption is discussed in Sect. 6.

The boundary problem for the system (3) was solved numerically using the pre-calculated profile of Pedersen permittivity $\varepsilon_{\perp}(z)$. This solution determines leaking Alfvén modes and their complex eigenfrequencies. The boundary condition at the bottom of Elayer (at $x^{3}=h=80 \mathrm{~km}$ ) is $b_{2}=0$, which denotes the non-penetration of wave fieldaligned current into a low-conductive atmosphere.

The boundary condition imposed at the upper IAR interface with the magnetosphere at $x^{3}=H$, demands a special consideration. Strictly speaking, the ionospheric plasma and geomagnetic field $\mathbf{B}_{0}$ are inhomogeneous, so the WKB-approximation for the system (3) cannot be applied. That is why the solution at $x^{3} \geq H$ as a running up harmonic wave $\propto \exp \left(i k_{3} x^{3}\right)$ implicitly assumes a "break" of the Alfvén velocity profile $V_{A}\left(x^{3}\right)$ at $x^{3}=H$. The neglect of this peculiarity may distort the spectral pattern of eigenoscillations. To set a correct boundary emission condition at $x^{3}=H$ one should apply a smoothing of the plasma parameters with some model. We have used the well-known model (Greifinger and Greifinger 1968)

$$
V_{A}\left(x^{3}\right)=V_{0}\left[\delta^{2}+\exp \left(-x^{3} / h\right)\right]^{-1 / 2},
$$

where parameter $\delta \ll 1$ determines the Alfvén velocity contrast between the F-layer and upper ionosphere. For this model the system (3) has an analytical solution via Bessel's functions. The profile of Alfvén velocity (5) at $x^{3} \rightarrow \infty$ tends to the constant value $V_{\infty}=V_{0} / \delta$, which enables us to use the asymptotic as a combination of running up and going down waves $\propto \exp \left( \pm i k_{3} x^{3}\right)$, where $k_{3}=\omega\left(V_{\infty} \sin I\right)^{-1}$. With the adjustment of the parameters $V_{0}, \delta$, and $h$, a good agreement of the modeling profile $V_{A}\left(x^{3}\right)$ with the realistic IRI-derived profile at $x^{3} \leq H$ can be achieved in a wide range of altitudes (see example in Fig. 2). The replacement of realistic profile at $x^{3} \geq H$ to the modeled one (5) gives us a possibility to use in this region an exact solution of the system (3), and therefore to reformulate the emission condition at $x^{3} \rightarrow \infty$ to the altitude $x^{3}=H$.

\section{Numerical modeling results}

Here we provide the results of the numerical modeling. The calculations were made for the conditions corresponding to middle latitude station Moshiri in the Northern hemisphere with geographical coordinates $44.37^{\circ} \mathrm{N}, 142.27^{\circ} \mathrm{E}$, the corresponding dip-angle is $I=$ $58.6^{\circ}$ (Shiokawa et al. 2010). The vertical component of the geomagnetic field $B_{0 z}=$ $41,200 \mathrm{nT}$, the parameter of geomagnetic field line $L=1.6, \mathrm{LT} \simeq \mathrm{UT}+9$. The ground conductivity is high, $\sigma_{g}=0.001 \mathrm{~S} / \mathrm{m}$, and corresponds to the skin-depth $\delta_{g} \simeq 16 \mathrm{~km}$ for $f=1 \mathrm{~Hz}$. The atmospheric conductivity is supposed to be $\sigma_{a}=5 \cdot 10^{-13} \mathrm{~S} / \mathrm{m}$.

Here we present the typical features of the ionospheric and IAR parameters and their diurnal variations for Sep. 19, 2008 (day number 263). For each UT the IRI profile has 


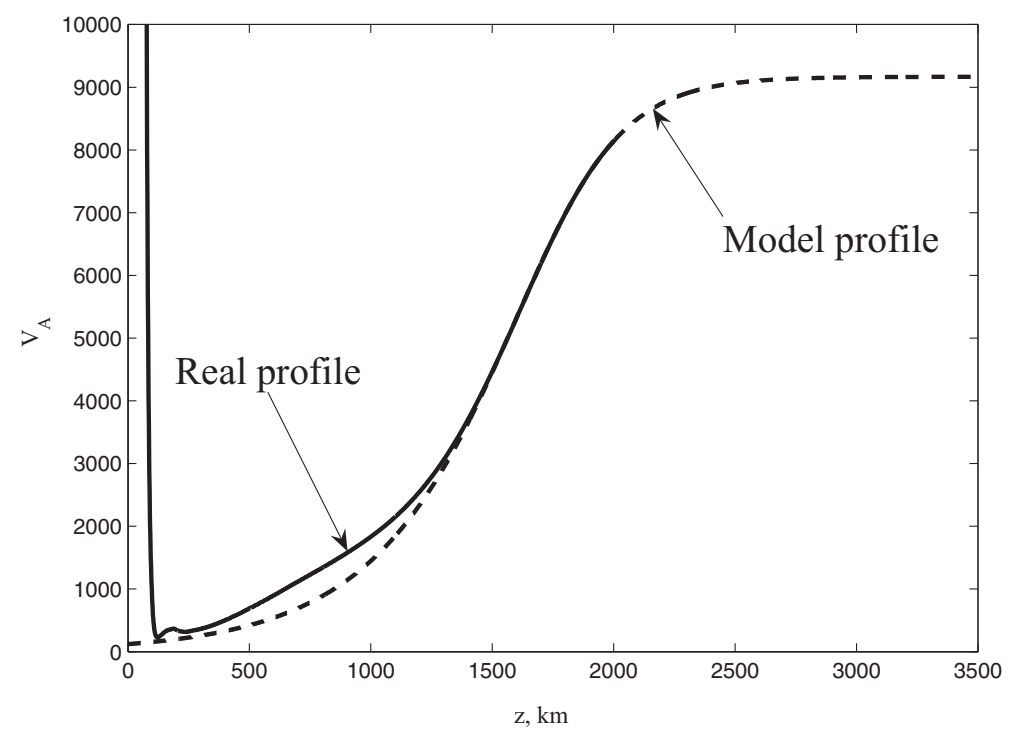

Fig. 2 The modeling profile $V_{A}(z)$ and the realistic IRI-derived profile (UT $=04$ )

been constructed for the corresponding 12-months running mean solar Wolf number $R z_{12}=7.7$.

\subsection{Diurnal variations of ionospheric parameters}

The height-integrated Pedersen conductance $\Sigma_{P}$ (Fig. 3) varies in the range from $\sim 7 \mathrm{~S}$ during daytime 07-17 LT (22-08 UT) to $\sim 0.1 \mathrm{~S}$ during nighttime 20-04 LT (11-19 UT). Diurnal variations of the characteristic Alfvén wave conductance of the ionospheric plasma above the E-layer $\Sigma_{A}=1 /\left(\mu_{0} V_{A}\right)$, determined by Alfvén velocity $V_{A}^{(m)}$ at the maximum of F-layer $z=h_{m} F 2$, are not very pronounced, and keep at the level about $2 \mathrm{~S}$ throughout the day.

Fig. 3 Diurnal variations of the height-integrated conductance $\Sigma_{P}$ and wave conductance $\Sigma_{A}$ derived from the plasma density of the F-layer maximum

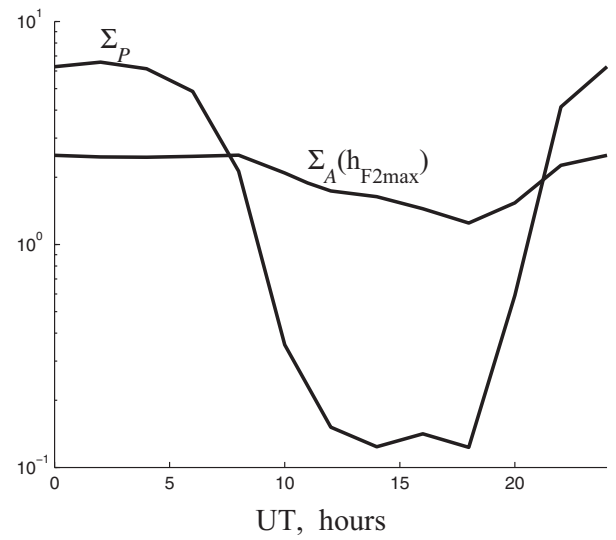


Profiles of the basic ionospheric and MHD parameters along a field line: electron plasma density $N_{e}(s)$ (Fig. 4, upper panel) and Alfvén refractive index $n_{A}(s)$ (Fig. 4, bottom panel), are shown for various UT. Though the decrease rate of $N_{e}$ with altitude is nearly the same at all UT, the gradient of $n_{A}(s)$ is more steep during night hours 20-04 LT (UT = 11-19) than during daytime hours 07-17 LT (UT = 22-08). This difference is due to the contribution of heavy ions $\left(\mathrm{O}^{+}, \mathrm{NO}^{+}\right)$into the content of the upper ionosphere, and consequently into the effective Alfvén velocity $V_{A}(s)$.

During nighttime a valley is formed in $n_{A}(s)$ profile between F-layer and E-layer at altitudes $\sim 140-200 \mathrm{~km}$. Ermakova et al. (2007) associated the eigenmode of this valley with wideband spectral peak around $\sim 6 \mathrm{~Hz}$, which they named sub-IAR. Here we do not consider this feature.

\subsection{Vertical structure of electromagnetic fields}

As an example we consider the wave vertical structure of second harmonic $(n=2)$ during daytime UT $=04$ (Fig. 5) and nighttime UT $=16$ (Fig. 6) hours. Amplitude $\left|b_{2}(z)\right|$ and $\left|e_{1}(z)\right|$ (Fig. 5, left-hand panel) variations correspond to the standing mode structure inside IAR $(z<1200 \mathrm{~km})$ : antinodes of magnetic component coincide with nodes of electric component.

The ratio between electric and magnetic component, or impedance $Z(z)=\mu_{0} e_{1} / b_{2}$ (Fig. 5, middle panel), above the IAR $(z>1400 \mathrm{~km})$ has value corresponding to the travelling Alfvén wave, $|Z| \rightarrow\left|Z_{A}\right|=\mu_{0} V_{A} \sin I$. Inside IAR, $|Z|$ drops to a lower value.

The phase shift between electric and magnetic component $\psi(z)=\operatorname{Arg}\left(e_{1} / b_{2}\right)$ (Fig. 5, right-hand panel) reveals different wave structures inside and above IAR. At $z>1400 \mathrm{~km}$

Fig. 4 Profiles of ionospheric and MHD parameters along a field line, as a function of the distance $s$ measured from the bottom of the ionosphere: electron plasma density $N_{e}(s)$ (top panel) and Alfvén refractive index $n_{A}(s)$ (bottom panel) for various values of UT

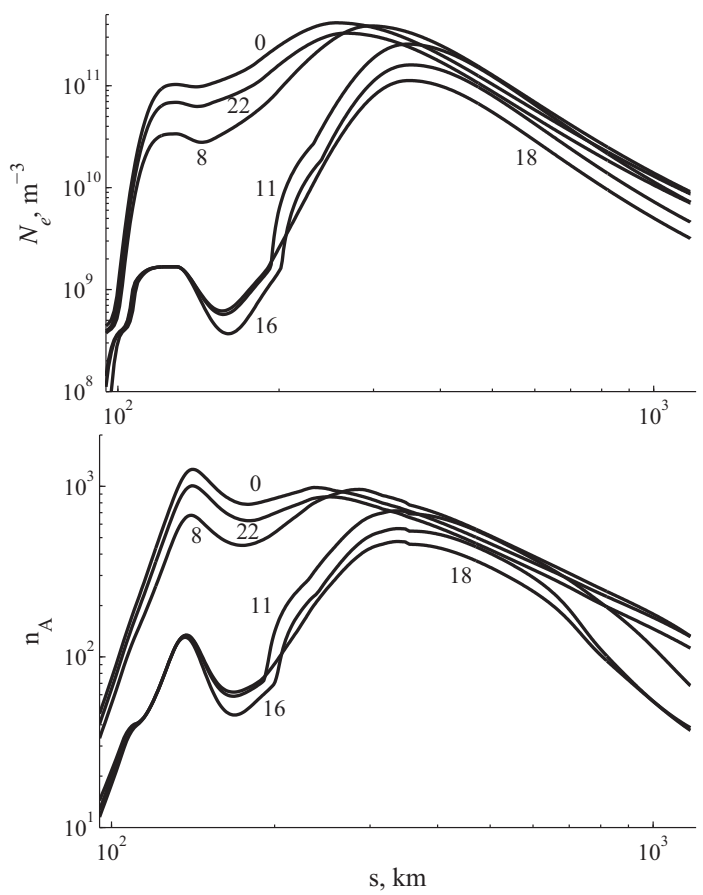



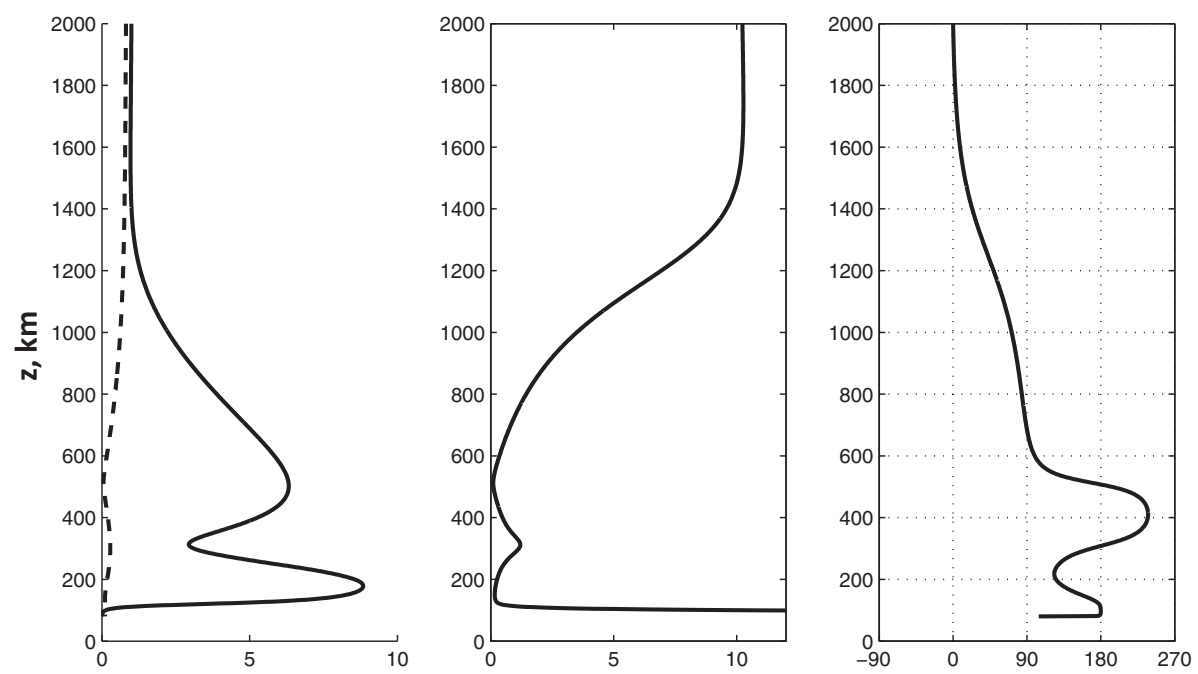

Fig. 5 The vertical wave structure of the second harmonic $(n=2)$ during daytime hours $(\mathrm{UT}=4)$. Lefthand panel amplitude profile $\left|b_{y}(z)\right|, \mathrm{nT}$, (solid line) and $\left|E_{x}(z)\right|, \mathrm{mV} / \mathrm{m}$, (dashed line); the impedance profile $|Z(z)|$, Ohm, (middle panel); and the vertical structure of the phase shift between electric and magnetic component $\psi(z)$, degrees (right-hand panel)
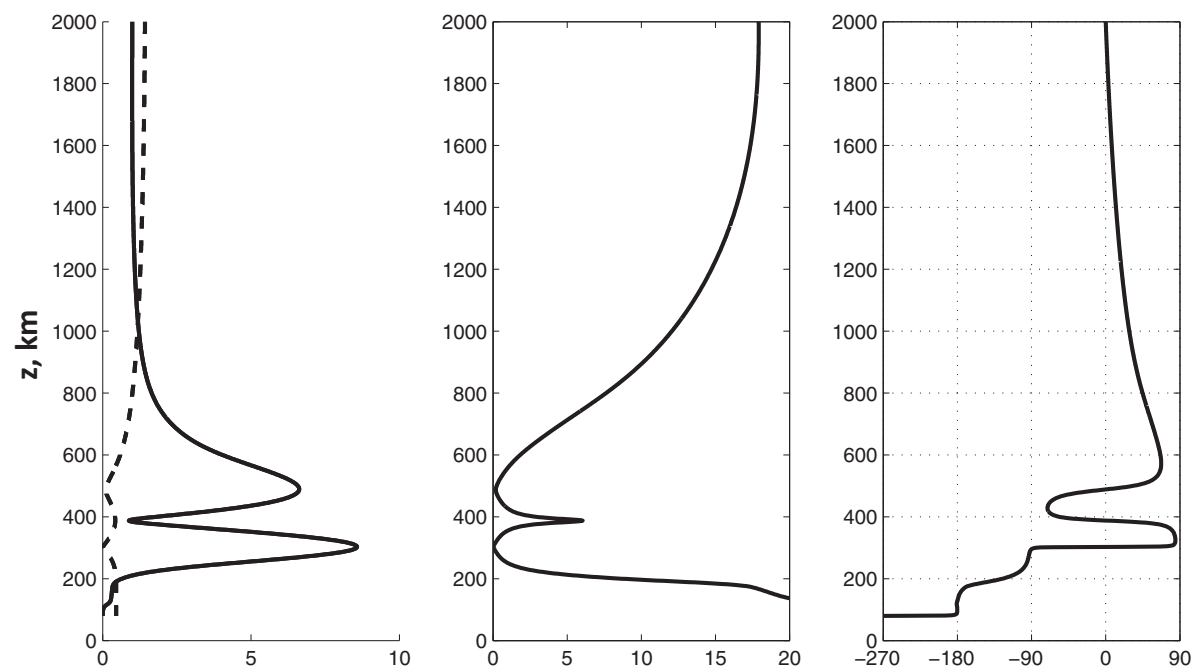

Fig. 6 The vertical structure of the second harmonic $(n=2)$ during nighttime hours $(\mathrm{UT}=16, \mathrm{LT}=01)$ in the same format as Fig. 5

$\psi \rightarrow 0$, which is a feature of running wave, leaking from the IAR. At $z<1200 \mathrm{~km}$ $\psi \rightarrow \pi / 2$, which is typical for a standing wave structure. Thus, the altitude $\sim 1400 \mathrm{~km}$ may be considered as a reflection point of Alfvén wave. The phase shift $\psi(z)$ experiences a jump about $\pi$ upon crossing the mode node or anti-node. 
During nighttime (Fig. 6) the IAR eigenmode is shifted to lower altitudes as compared with daytime. The wave impedance increases upon moving to lower altitudes (Fig. 6, medium panel). The phase shift between electric and magnetic component is more evident during nighttime than during daytime (Fig. 6, right-hand panel).

\subsection{Spectral and energy characteristics of the IAR}

Diurnal variations of the first four IAR harmonics eigenfrequencies $f_{n}(t)$ and Q-factors $Q_{n}(t)$ are shown in Fig. 7. As expected, the IAR eigenfrequencies (upper panel) during daytime are lower than those for the nightside ionosphere due to a higher ionospheric plasma density. All harmonics gradually increase (e.g., $f_{1}$ from 0.3 to $1 \mathrm{~Hz}$ ) till $18 \mathrm{UT}$ (LT=3), and rapidly decrease after this time.

The Q-factor (Fig. 7, middle panel) is larger during nighttime than during daytime. The most significant contrast is for lower harmonics $(n=1-2)$. Thus, $Q_{1}>7$ during 11-18 UT with extreme $Q_{1} \approx 9$ at $14 \mathrm{UT}$, whereas the daytime values are $Q_{1} \sim 2$. Variations of Qfactor for higher harmonics $(n=3-4)$ are less significant: from $\sim 3-5$ during daytime up to $\sim 7$ during nighttime.

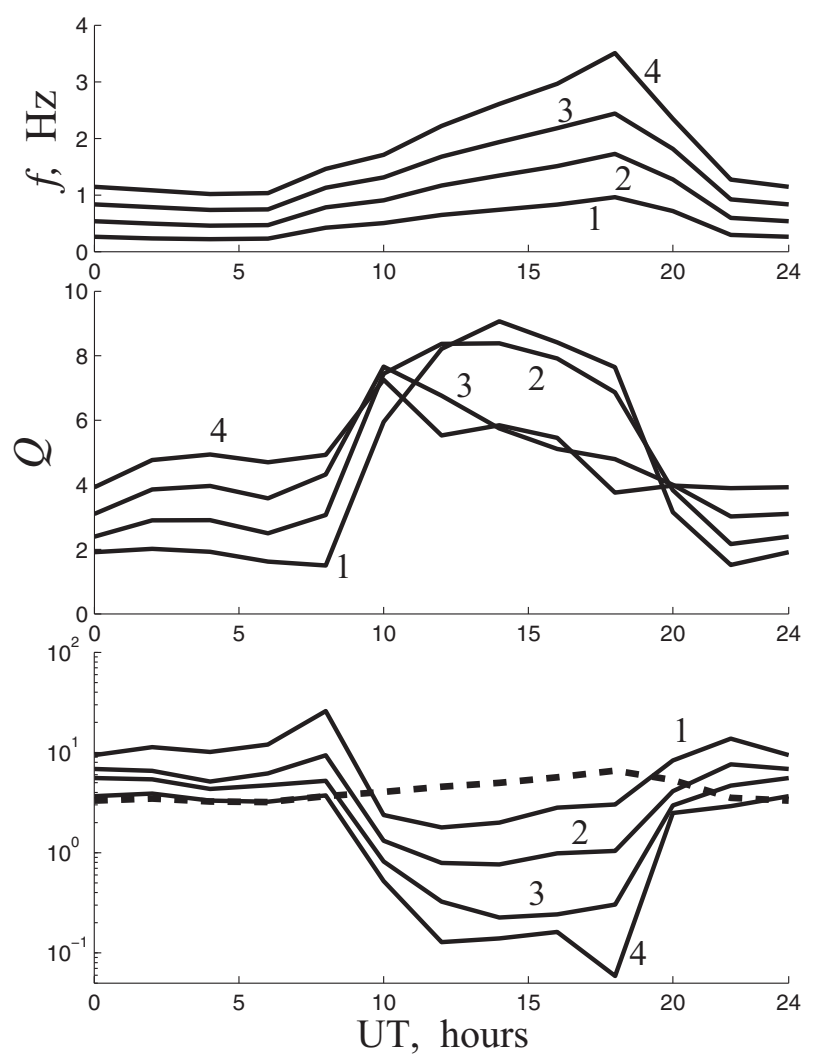

Fig. 7 Diurnal variations for 4 first IAR harmonics of eigenfrequencies $f_{n}$ (UT) (top panel), Q-factors $Q_{n}(\mathrm{UT})$ (middle panel), and Joule losses, $\mu \mathrm{Jm}^{-2} \mathrm{~s}^{-1}$, (bottom panel, solid lines); energy flux density at $2000 \mathrm{~km}$, $\mu \mathrm{Jm}^{-2} \mathrm{~s}^{-1}$, is shown with dashed line. The labels $1-4$ correspond to harmonic numbers, $\mathrm{LT} \simeq \mathrm{UT}+9$ 
The Q-factor is determined by two main energy loss processes: (a) leakage through the upper IAR boundary into the magnetosphere; and (b) Joule dissipation in the conductive layers of lower ionosphere. The height-integrated Joule dissipation $J=(1 / 2) \int_{0}^{H} \sigma_{P}\left|e_{1}\right|^{2} d z$ is shown in Fig. 7 (bottom panel) for each harmonic by solid line. This plot shows that for all harmonics the Joule dissipation is the dominating damping mechanism during daytime, whereas a leakage into the magnetosphere, characterized by the Poynting energy flux (shown with dashed line) becomes most significant during nighttime.

Figure 8 presents dependences on harmonic number $n$ of harmonic frequency $f_{n}$ (lefthand panel) and $Q_{n}$ (right-hand panel). During nighttime, UT $=11-19$, (dark dots) the frequencies $f_{n}$ are higher than during daytime, UT $=22-08$ (empty dots). The frequencies are nearly equidistant and linearly grow with $n$. This justifies the equidistant estimate of spectral scale $\Delta f$, made in WKB approximation (e.g., Potapov et al. 2014).

During daytime Q-factors are rather low, e.g. $Q_{1} \simeq 2$, but noticeably increase with $n$. During nighttime (dark filled circles) Q-factors are higher, but decrease somewhat with $n$ from $\sim 8-9$ to $\sim 6$. On opposite, during daytime (empty circles) the Q-factor is low for lowest harmonics, but it increases till $\sim 3-4$ for higher harmonics $(n>3)$. In other words, during nighttime lower harmonics $n=1-2$ are better trapped in the IAR than higher harmonics $n=3-4$. In this aspect, the numerical modeling results differ from the
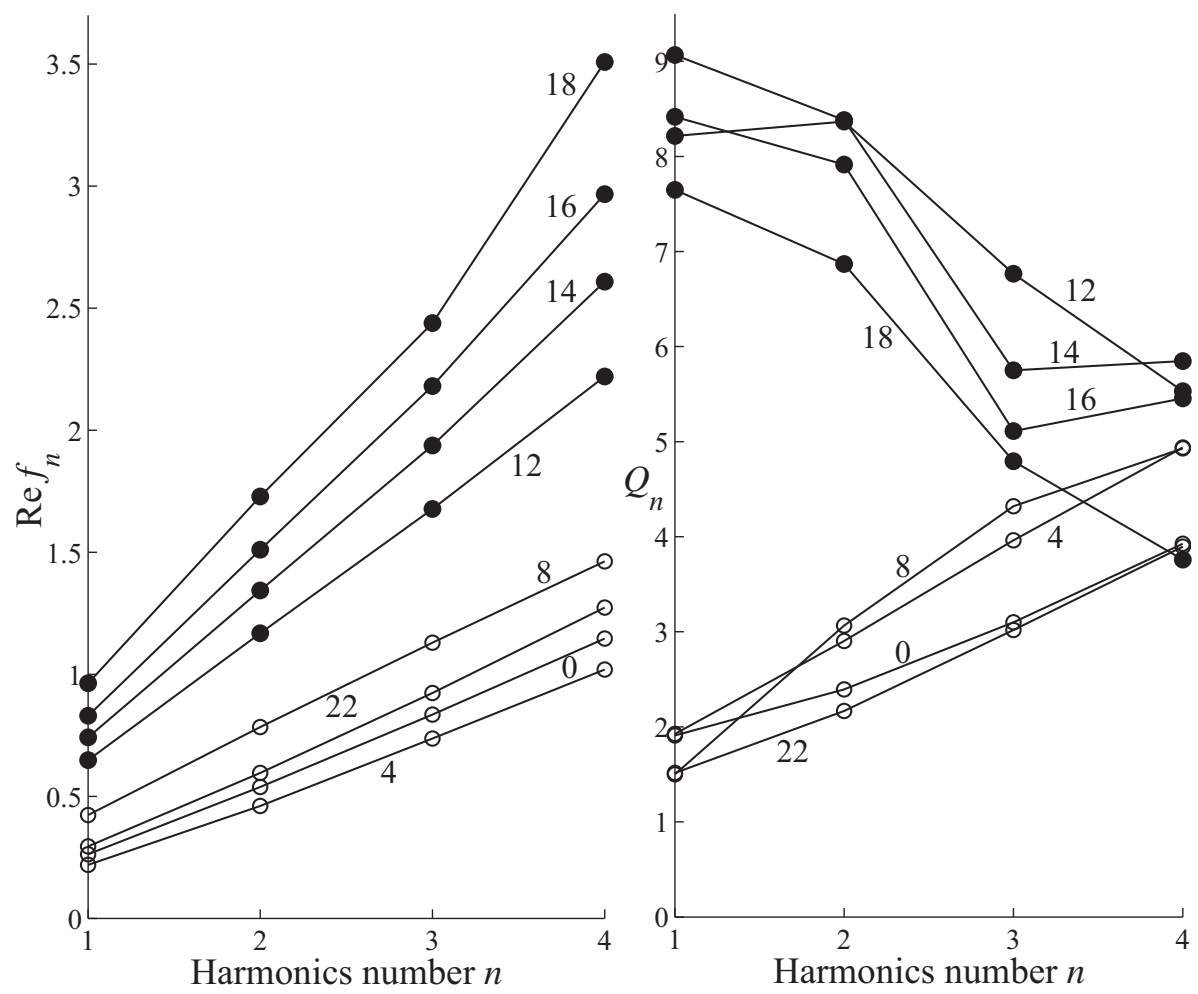

Fig. 8 Dependences of the IAR eigen frequencies $\operatorname{Re} f_{n}$ (left-hand panel) and Q-factors $Q_{n}$ (right-hand panel) on harmonic number $n$. Filled circles correspond to night hours, and empty circles correspond to daytime. UT times are indicated near each curve $(\mathrm{LT} \simeq \mathrm{UT}+9)$ 
predictions of the analytical model (Polyakov and Rapoport 1981), which claimed that Q-factor is to be the same for all harmonics.

\section{Diagnostics of the upper ionosphere with the use of IAR spectral features}

The spectral features of IAR-associated structure observed on the ground can be used for ground-based monitoring of the nighttime F-layer peak plasma density $N_{m}$. Diurnal evolution of TEC is qualitatively similar to $N_{m}$ variations and can be determined from ground magnetometer data, too (Fig. 9).

The correspondence between $\Delta f$ and $N_{m}$ during nighttime hours 06-16 UT (15-01 LT) when IAR signatures are evident, is well approximated on a $\log -\log$ scale by a linear fit with coefficient $\alpha=-0.97 \approx-1$ (Fig. 10, bottom panel). Therefore, the relationship between them can be modeled as

$$
N_{m}\left[\mathrm{~m}^{-3}\right]=1.2 \cdot 10^{5}\left(\frac{B_{0 z}}{41,200}\right)(\Delta f[\mathrm{~Hz}])^{-1} .
$$

This relationship has been parameterized by geomagnetic field and inclination, as $f_{A} \propto \int d s / V_{A} \propto \int d z / B_{0} \sin I \propto 1 / B_{0 z}$, where $B_{0 z}$ is the vertical component of geomagnetic field in an observation site.

The relationship between $\Delta f$ and TEC during night hours, 06-16 UT(15-01 LT), when IAR signatures are evident, can be examined in a similar way. The dependence between TEC and $\Delta f$ in log-log coordinates is nearly linear (Fig. 10, top panel). The factor in the linear regression $\alpha=-0.95$ is also very close to 1 . Therefore, the dependence of TEC (measured in TECU $=10^{16} \mathrm{~m}^{-2}$ ) on $\Delta f$ is well approximated by formula

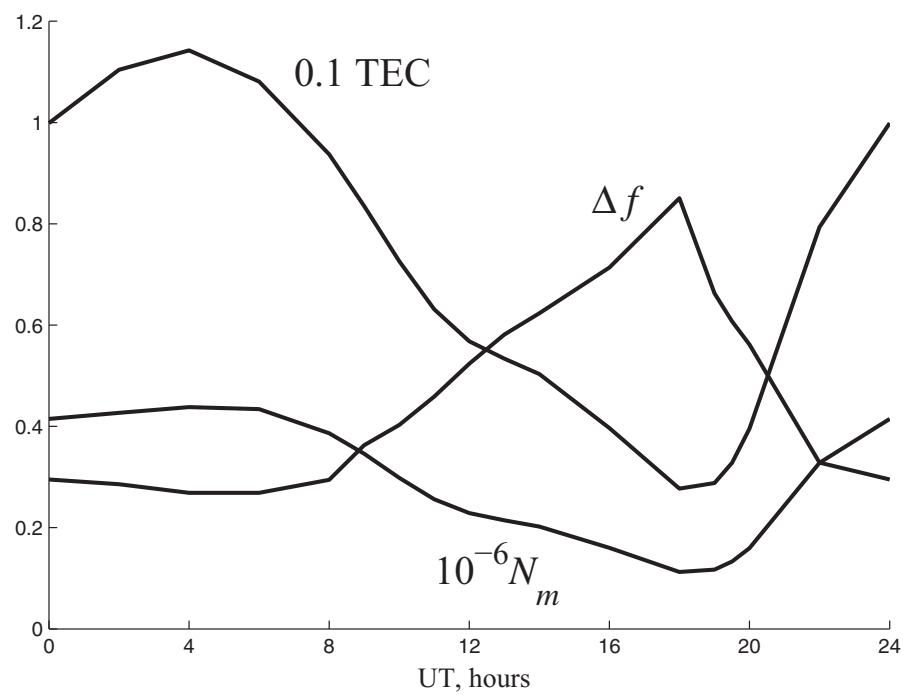

Fig. 9 Diurnal evolution of the F-layer peak plasma density $N_{m}$, TEC, and the IAR spectral scale $\Delta f$ 
Fig. 10 The correspondence during nighttime hours 06-16 UT (15-01 LT) between a $\Delta f$ and $N_{m}$, and $\mathbf{b} \Delta f$ and TEC
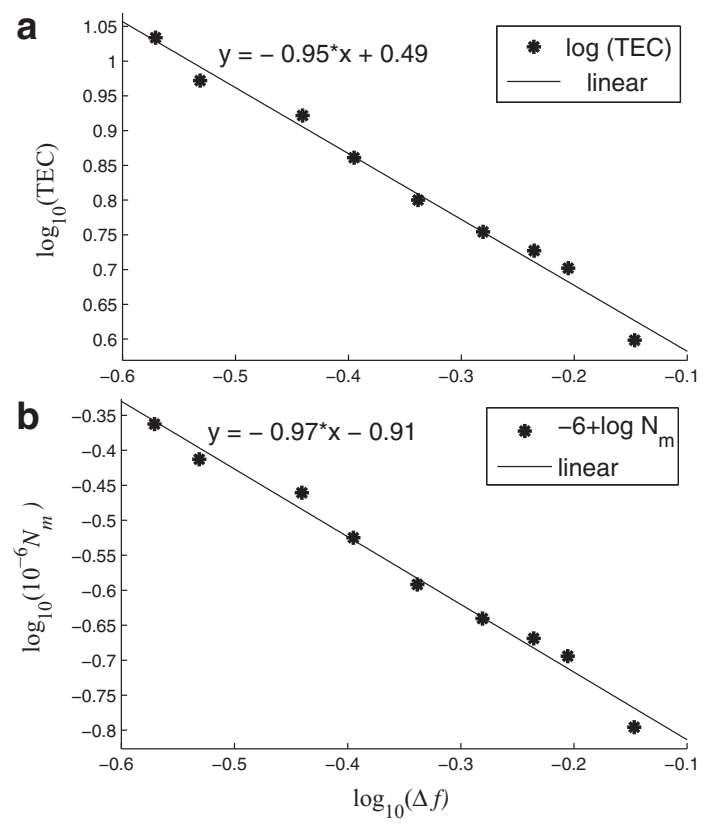

$$
T E C[\mathrm{TECU}]=3.1\left(\frac{B_{0 z}}{41,200}\right)(\Delta f[\mathrm{~Hz}])^{-1} .
$$

We compare our modeling results with the spectral relationships from Alpatov et al. (2005) numerical model based on approximation of $n_{A}(z)$ and a perfectly reflecting E-layer. According to these calculations, dependence of resonant frequencies on harmonic number $n$ was approximated as $\omega_{n} / \Omega_{A} \simeq(2 n+1) / 3$, whereas the interval between eigenfrequencies was $\Delta \omega / \Omega_{A} \simeq 2 / 3$, where $\Omega_{A}$ is the resonator fundamental frequency. In our model the ratio $\Delta \omega / \Omega_{A}=1.18$ for $\mathrm{UT}=4$, and 0.86 for $\mathrm{UT}=16$, which is different from 0.66 in (Alpatov et al. 2005).

\section{The validity of the decoupled Alfvénic model}

Let us estimate when the neglect of mode coupling owing to the Hall conductivity is possible for the determination of the IAR eigenfunctions. Here we show that this approximation is justified for a sufficiently small transverse wave scales (or large wave numbers $k$ ).

For such estimate we use a simplified model with an analytical solution. The Hall conductance is assumed to be concentrated in thin layer (E-layer) located at altitude $h$ above the Earth's surface $\sigma_{H}(z)=\Sigma_{H} \delta(z-h)$, where $\delta$ is the delta-function. Below this thin layer is a homogeneous non-conductive space with the dielectric permeability $\varepsilon_{a}=\varepsilon_{0}$ (atmosphere), and above there is a plasma space characterized by Alfvén velocity $V_{A}$. The complex Pedersen conductivity is distributed among the E-layer and upper space above it 
as follows $\sigma_{P}(z)=\Sigma_{P} \delta(z-h)+\sigma_{P}^{(m)}(z)$, where $\sigma_{P}^{(m)}(z)=0$ at $z<h$ and $\sigma_{P}^{(m)}(z)=$ $-i \omega \sqrt{\varepsilon_{0} / \mu_{0}} V_{A}^{-1}$ at $z>h$.

Within the frameworks of this model the system (2) decouples into sub-systems for Alfvén and FMS waves above the E-layer. These modes are coupled in the thin layer at $z=h$ with a high anisotropic conductivity. This coupling is described by the heightintegrated across E-layer Eq. (2). These equations yield the continuity of transverse electric components, $e_{1,2}(h-0)=e_{1,2}(h+0)$, and determine the jump of magnetic field components

$$
\begin{gathered}
b_{1}^{+}-b_{1}^{-}=-\mu_{0} \Sigma_{H}(\sin I)^{-1} e_{1}+\mu_{0} \Sigma_{P} e_{2}, \\
b_{2}^{+}-b_{2}^{-}=-\mu_{0} \Sigma_{P}(\sin I)^{-2} e_{1}-\mu_{0} \Sigma_{H}(\sin I)^{-1} e_{2},
\end{gathered}
$$

where $b_{1,2}^{ \pm}=b_{1,2}(h \pm 0)$.

The Alfvén wave interaction with the ionosphere is determined by the admittance of Alfvén waves, that is the ratio $b_{2}^{+} / e_{1}$ between magnetic and electric components at $z=h+0$. In general, this admittance depends on the Hall conductance $\Sigma_{H}$. Let us find the condition when this dependence becomes insignificant. The proper admittances are found from the Maxwell's equations for TM (magnetic) mode in the atmosphere with boundary condition $e_{2}(0)=0$ at ideally conductive Earth's surface and for FMS mode in the upper space $z>h$ with the emission condition at $z \rightarrow \infty$ as follows

$$
\begin{aligned}
b_{1}^{-} / e_{2}=i \omega^{-1} \kappa_{a} \cot \left(\kappa_{a} h\right) & \approx i \omega^{-1} k \operatorname{coth}(k h), \\
b_{1}^{+} / e_{2}=-\omega^{-1} \kappa_{m} & \approx-i \omega^{-1} k,
\end{aligned}
$$

where $\kappa_{a}=\left(k_{0}^{2}-k^{2}\right)^{1 / 2}, \kappa_{m}=\left(k_{A}^{2}-k^{2}\right)^{1 / 2}$, and $k_{0}=\omega / c$ is the vacuum wave number. The approximate relationships (8) have been derived under the assumption that realistic wave transverse scale is not very large, namely $k \gg k_{A}$ and $k \gg k_{0}$. The relationships (8) and (6) enable us to exclude $e_{2}$ from (7). Using the condition of current non-penetration into the atmosphere $b_{2}^{-}=0$ we obtain from (7) the sought-for admittance-type condition for Alfvén waves

$$
b_{2}^{+}=-\mu_{0} Y_{A} e_{1}
$$

Here $Y_{A}$ is the ionospheric surface admittance for Alfvén waves

$$
Y_{A}=\Sigma_{P}(\sin I)^{-2}\left(1+\frac{\left(\Sigma_{H} / \Sigma_{P}\right)^{2}}{1+i k d_{P}}\right),
$$

where $d_{P}=[1+\operatorname{coth}(k h)] /\left(\mu_{0} \omega \Sigma_{P}\right)$ is the scale determined by the Pedersen conductance. The second term in (9) corresponds to the influence of the Hall conductivity.

Impact of the Hall conductivity on IAR resonance frequencies can be estimated from its influence on the reflection coefficient $R_{A}$ of Alfvén waves from the E-layer. The reflection of Alfvén wave from the ionosphere with the surface admittance $Y_{A}$ is characterized by the coefficient (e.g., Fedorov et al. 2001)

$$
R_{A}=\frac{Y_{A}-\Sigma_{A}}{Y_{A}+\Sigma_{A}} .
$$

Correspondingly, the reflection coefficient from the ionosphere with a vanishing Hall conductivity is 


$$
R_{A}^{(0)}=\frac{Y_{A}^{(0)}-\Sigma_{A}}{Y_{A}^{(0)}+\Sigma_{A}},
$$

where $Y_{A}^{(0)}=\Sigma_{P}(\sin I)^{-2}$ is obtained from (9) under $\Sigma_{H}=0$.

The influence of the Hall conductivity on the reflection coefficient $R_{A}$ can be neglected if the relative error $\delta_{H}$ is small, namely

$$
\delta_{H}=\left|R_{A} / R_{A}^{(0)}-1\right| \ll 1 .
$$

Using Eqs. (9), (10), and (11) we find the parameter $\delta_{H}$ explicitly

$$
\delta_{H}=\frac{2 S}{|1-S|}\left[(1+U)^{2}+\left(k d_{P} U\right)^{2}\right]^{-1 / 2},
$$

where dimensionless functions $S=\Sigma_{A} / Y_{A}^{(0)}=\left(\Sigma_{A} / \Sigma_{P}\right) \sin ^{2} I$, and $U=\left(\Sigma_{P} / \Sigma_{H}\right)^{2}(1+S)$. For a fixed $\Sigma_{P}$, the magnitude of $\delta_{H}$ decreases monotonically upon $\Sigma_{H}$ decrease ( $U$ growth), or upon $k$ increase.

For typical mid-latitude dayside conditions $\left(\Sigma_{P} \sim \Sigma_{H} \gg \Sigma_{A} \sim 1 \mathrm{~S}\right)$ the maximal value of $\delta_{H}$ is small. Indeed, because $S \ll 1$, therefore $\delta_{H}(k, U)<\delta_{H}(k=0, U=0)=$ $2 S|1-S|^{-1} \ll 1$, so the condition (12) is satisfied universally, irrespectively of $\Sigma_{H}$ or $k$ values. On the contrary, for typical mid-latitude nightside conditions $S \gg 1$ (e.g., $\left.\Sigma_{P} \ll 1 \mathrm{~S}, \Sigma_{A} \sim 1 \mathrm{~S}\right)$, so the value of $\delta_{H}(k=0, U=0)=2 S|1-S|^{-1} \sim 2$ is finite. Thus, the condition (12) is satisfied only for a sufficiently small Hall conductance $\Sigma_{H}$, whereas the smaller $k$ the smaller conductance ratio $\Sigma_{H} / \Sigma_{P}$ is necessary. For example, let $\Sigma_{P}=0.14 \mathrm{~S}$ and $k=10^{-1} \mathrm{~km}^{-1}, 10^{-2} \mathrm{~km}^{-1}$, and $10^{-3} \mathrm{~km}^{-1}$, then the Alfvénic decoupled model is valid for $\Sigma_{H} / \Sigma_{P} \ll 25,9$, and 6 , correspondingly.

The estimate (13) is more correct and less strict (though less elegant) than the condition of the Hall effect neglect from (Lysak and Yoshikawa, 2013) $k \gg\left(\Sigma_{H} / \Sigma_{P}\right)^{2} \mu_{0} \omega \Sigma_{P}$. However, the simple estimate (13) cannot be used when IAR frequency and the FMS waveguide frequency approach each other under a certain $k$.

These analytical estimates have been validated by numerical modeling. The numerical solution of the coupled system (2), corresponding to a 4th order differential equation, is considerably more complicated than the analysis of the decoupled Alfvénic mode (3), corresponding to a 2 nd order differential equation. The Eqs. (3) remain approximately valid for non-vanishing $k_{2} \neq 0$, within small-scale approximation in radial direction, $\left|k_{2}\right| \ll\left|k_{1}\right|$. The possible error upon neglect of the mode coupling owing to the Hall conductance has been estimated with more advanced numerical modeling, based on the system of coupled Eq. (2). The dependence of spectral characteristics of the fundamental $(n=1)$ IAR harmonic on wave number $k$ was calculated for night hour (16 UT) (Fig. 11). For this time interval the IRI model gives $\Sigma_{P}=0.14 \mathrm{~S}$ and $\Sigma_{H}=0.16 \mathrm{~S}$. The Alfvénic decoupled model results correspond to the case $\Sigma_{H}=0$ (solid line). In this case the eigenfrequency and Q-factor practically do not depend on $k$. Insignificant changes of $\operatorname{Re} f_{1}$ and $Q_{1}$ about $0.01 \%$ are caused by an influence of atmospheric and ground conductivities. For a coupled mode (dashed line), under given $\Sigma_{H}$ and $\Sigma_{P}$, the spectral parameters $\operatorname{Re} f_{1}$ and $Q_{1}$ deviate from their approximate values just slightly, $\sim 0.2 \%$. The largest variations occur for large-scale modes with $k \simeq 10^{-3}-10^{-2} \mathrm{~km}^{-1}$.

However, this example does not imply that the Hall conductivity may be always neglected in IAR studies. Let us consider the dependence of the IAR spectral parameters 
Fig. 11 The dependence of spectral characteristics of the fundamental $(n=1)$ IAR harmonic on wave number $k$ for night hour (16 UT)

$\left(\Sigma_{P}=0.141 \mathrm{~S}, \Sigma_{H}=0.158 \mathrm{~S}\right)$.

The Alfvénic model results corresponds to the case $\Sigma_{H}=0$ (solid line), the coupled mode model results are shown with dashed line

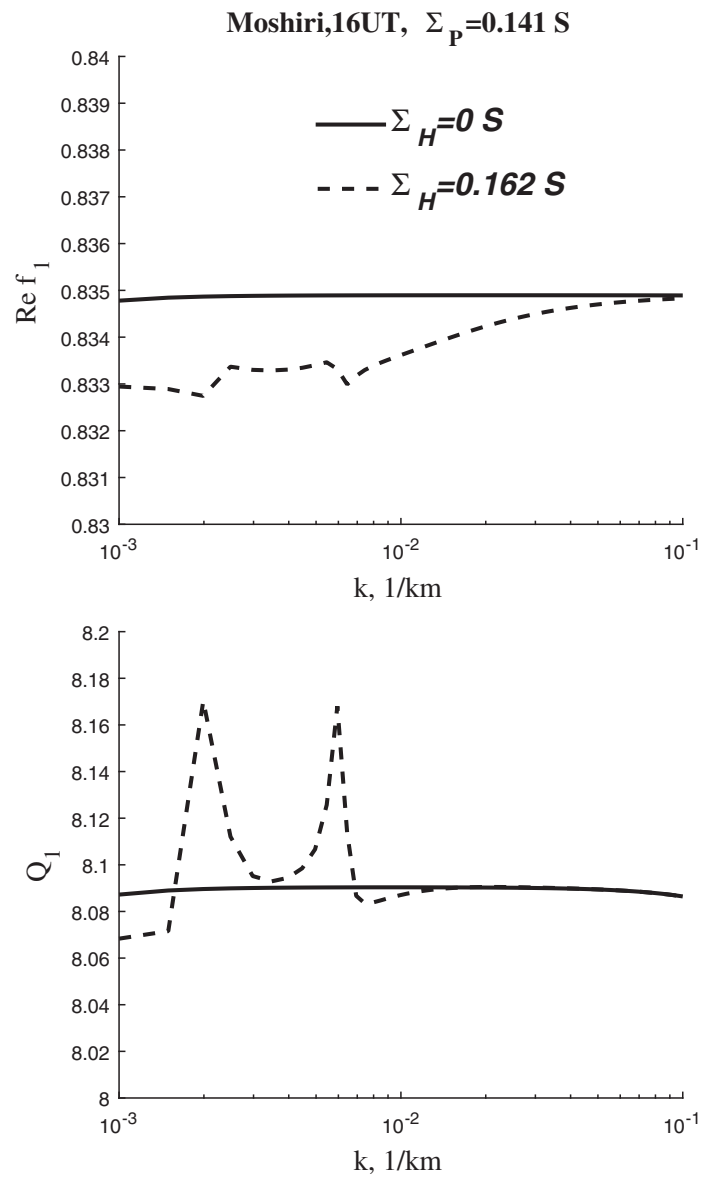

on $\Sigma_{H}$ for a fixed $\Sigma_{P}$. For that we have modified the IRI-derived Hall conductivity $\sigma_{H}^{(0)}$, multiplying it by a factor $\alpha$, that is $\sigma_{H}=\alpha \sigma_{H}^{(0)}$. Figure 12 shows the dependence of spectral parameters on the resultant ratio $\Sigma_{H} / \Sigma_{P}$ for 3 different values of $k$. While for small scale waves with $k=10^{-1} \mathrm{~km}^{-1}$ the IAR spectral parameters virtually do not depend on the ratio $\Sigma_{H} / \Sigma_{P}$, for larger scale waves, corresponding to $k=10^{-2} \mathrm{~km}^{-1}$ and $10^{-3} \mathrm{~km}^{-1}$, this influence becomes more significant. For $\Sigma_{H} / \Sigma_{P} \sim 2$ the influence of mode coupling is still weak, but when $\Sigma_{H} / \Sigma_{P} \rightarrow 10$ frequency $\operatorname{Re} f_{1}$ decreases by 20 and $25 \%$, and $Q_{1}$ increases by 17 and $22 \%$. The numerical calculation results presented in Fig. 12 confirm the condition (12), (13) when the simplified Alfvénic model (3) can be applied for the description of the IAR spectral parameters.

However, this condition has been derived and calculated for the fundamental IAR harmonic. For higher harmonics this condition may be insufficient. For example, calculations for nighttime conditions (16 UT) of the IAR spectral parameters $f_{4}$ and $Q_{4}$ for the 4th harmonic (Fig. 13) show that deviation between the full and approximate models is more significant than for the fundamental mode (Fig. 12). In general, numerical modeling showed that for the first 4 IAR harmonics for a typical mid- and low latitude ionosphere, 
Fig. 12 The dependence of spectral parameters eigenfrequency $f_{1}$ (upper panel) and quality factor $Q_{1}$ (bottom panel) of the fundamental IAR mode, on the ratio $\Sigma_{H} / \Sigma_{P}$ for three different values of $k$ (shown in the legend)

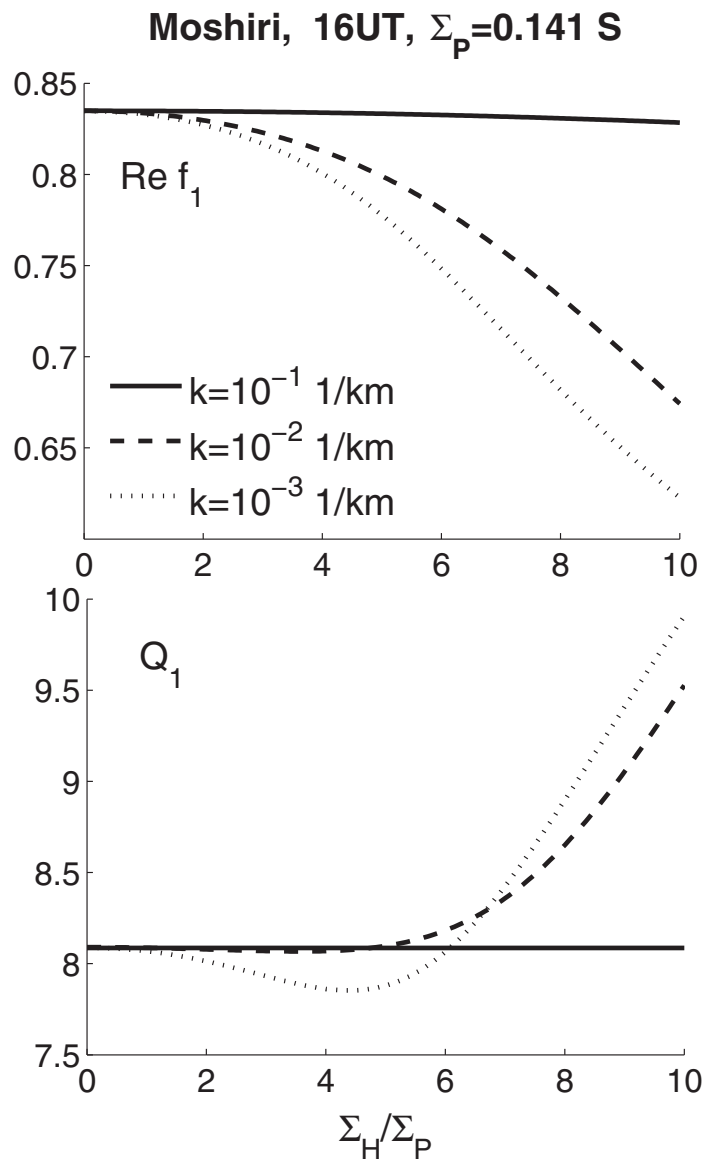

$\Sigma_{P} / \Sigma_{H} \leq 5$, the approximate Alfvénic model provides an error of the eigenfrequency and Q-factor determination less than $10 \%$ for wave scales from $10^{-3} \mathrm{~km}^{-1}$ up to small scales. At auroral latitudes, where the Hall conductance may be strongly enhanced as compared with the Pedersen conductance owing to electron precipitation, the validity of the decoupled mode approach is limited to a smaller scale.

\section{Discussion}

The diurnal variation of the IAR signature occurrence on the ground matches well the diurnal variations of Q-factor predicted by the model. In our model the Q-factor is determined by two main energy loss processes: (a) leakage through the upper IAR boundary into the magnetosphere; and (b) Joule dissipation in the conductive layers of lower ionosphere. During daytime Joule dissipation is the dominating damping mechanism, whereas a leakage into the magnetosphere becomes most significant during nighttime. 
Fig. 13 The same format, as Fig. 12, but for the 4-th IAR harmonic

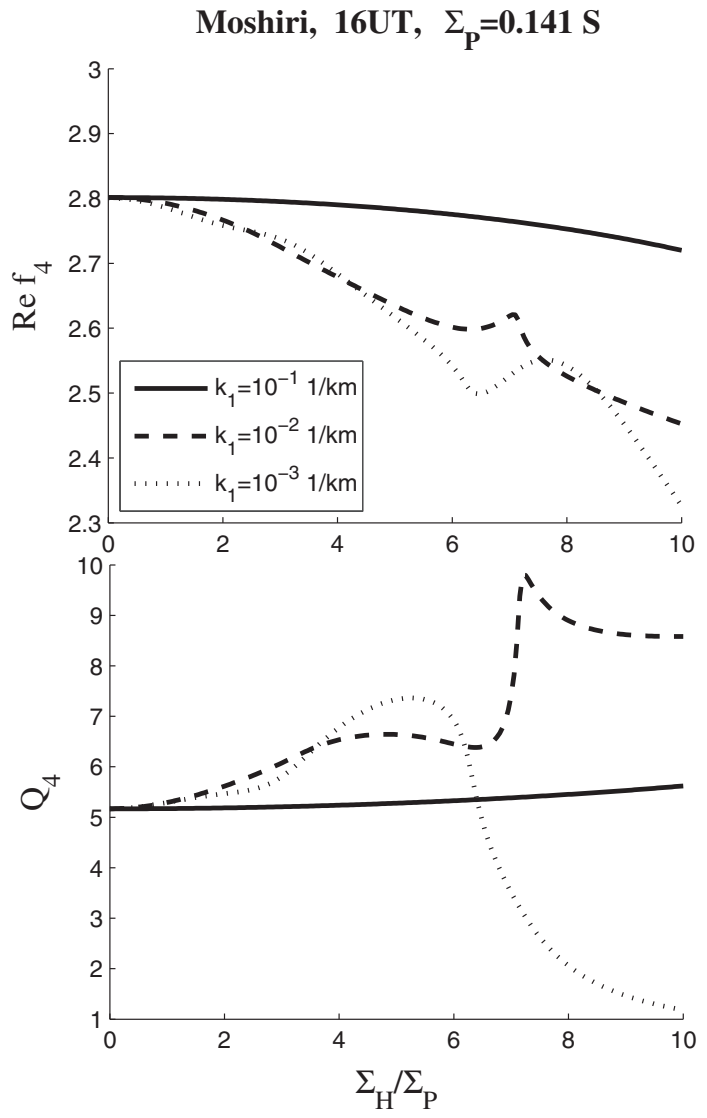

The Joule dissipation may explain a disappearance of the IAR signatures during periods with enhanced electron precipitation during magnetically disturbed times, because such precipitation increases the plasma density in the lower ionosphere, and consequently the Joule dissipation of IAR modes.

Even if an initial IAR driver is linearly polarized, upon propagation across the realistic ionosphere owing to the mode coupling an Alfven wave becomes elliptically polarized. However, just to estimate the IAR eigenfrequencies the mode coupling effects can be neglected (even at altitudes where diagonal tensor elements $g \neq 0$ ) under the condition (12). In the numerical model presented, as in all other IAR models, a coupling of Alfvén and FMS modes owing to the Hall conductance was neglected. The possible error has been estimated with more advanced numerical modeling. This modeling showed that for the lowest 4 IAR harmonics the simplified Alfvenic model under $\Sigma_{P} / \Sigma_{H} \leq 5$ provides an error of eigenfrequency and Q-factor determination less than $10 \%$. Thus, the Alfvénic uncoupled model is adequate to describe spectral parameters of the IAR at middle and low latitudes excited by sources with scales up to $\sim k^{-1} \leq 10^{3} \mathrm{~km}$.

Though the mode coupling owing to the Hall conductivity can be neglected under the provided above approximation to estimate the IAR eigenfrequencies, the ground response to ionospheric Alfvén disturbance is due solely to the Hall conductance. In principle a 
ground response to an ionospheric Alfvén mode can be estimated in the following way: using the approximate equations (3) wave electric and magnetic fields in the ionosphere are found; then Hall current induced by wave electric field is calculated; and finally magnetic components of TE-mode in the atmosphere, as well as of FMS mode in the ionosphere, produced by this Hall current are estimated. However, these estimates are beyond the scopes of this paper. Very small wave structures in the ionosphere, corresponding to $k h \gg 1$, where $h \simeq 100 \mathrm{~km}$ is the height of the conductive E-layer, are nearly totally screened from ground magnetometers. Such small-scale Afvénic structures, which are closely associated with auroral electron precipitation, occur predominantly at auroral latitudes. Even smaller structures, about few $\mathrm{km}$, are to be severely absorbed by the collisional ionosphere (Lessard and Knudsen 2001).

The presented Alfven wave model is the basis for a complete description of the IAR eigenvalues. To interpret specific ground or satellite observations of a spectral structure one has to solve a problem on the excitation of the ionospheric resonator/waveguide by a given source with known frequency-spatial parameters. A numerical solution of such problem on the ionospheric cavity excitation by an atmospheric lightning discharge or a magnetospheric ULF noise will be presented elsewhere.

\section{Conclusion}

The term IAR is widely used nowadays to characterize a MHD system with a minimum of Alfven wave field-aligned distribution which can partially trap Alfven waves with certain frequencies an scales. Such systems have been found in the solar atmosphere, terrestrial ionosphere, and planetary environment. IAR can be excited by various mechanisms, and its characteristic signature-multi-band spectral structure, can be used for the spectroscopy of remote plasma objects. For such spectroscopy of the top-side terrestrial ionosphere we have developed a numerical model based on realistic profile of the plasma constituents. The model-derived relationships can be used to restore the plasma density in the maximum of the nightside F-layer or vertical TEC from data of ground magnetometer observations of the frequency scale $\Delta f$ in the IAR spectral pattern. The relevant relationships can be easily recalibrated for any other location with another latitude/inclination parameters. The eigenspectrum given by the numerical model is nearly equidistant, which simplifies the choice of frequency interval for an estimate of $\Delta f$. The model clearly presents a contribution of Joule dissipation and wave leakage into the dissipative properties of the IAR eigenmodes at different local times. Combination of these factors is shown to determine the occurrence of the IAR signatures during observations. The model predicts a vertical structure of IAR eigenmodes and their effective impedance ( $E$ / $B$ ratio) which can be used in low-Earth orbit observations to identify these modes. The widely used Alfvénic approach to the IAR modeling, as has been shown analytically and numerically, is valid at mid/low latitudes for wave transverse scales less than $\sim 10^{3} \mathrm{~km}$. At auroral latitudes, where the Hall/Pedersen conductance ratio may be strongly enhanced owing to electron precipitation, the validity of the decoupled mode approach is limited to a very small scale.

Acknowledgments This research is supported by grants from the Russian Fund for Basic Research 13-0512091 (EF, EE), 15-05-01814 (VP), and Program 22 of Russian Academy of Sciences (NM). The useful discussions with Shiokawa K. and helpful comments from both reviewers are appreciated. 


\section{References}

Alpatov VV, Deminov MG, Faermark DS, Grebnev IA, Kosch MJ (2005) Dynamics of Alfvén waves in the night-side ionospheric Alfvén resonator at mid-latitudes. Ann Geophys 23:499-507

Alperovich LS, Fedorov EN (2007) Hydromagnetic waves in the magnetosphere and the ionosphere., Astrophysics and Space Science LibrarySpringer Science \& Business Media, New York

Belyaev PP, Polyakov SV, Rapoport VO, Trakhtengertz VY (1987) Discovery of the resonance spectrum structure of atmospheric electromagnetic noise background in the range of short-period geomagnetic pulsations, Doklady Akad. Nauk SSSR 297:840-846

Belyaev PP, Polyakov SV, Rapoport VO, Trakhtengerts VYu (1989) Theory for the formation of resonance structure in the spectrum of atmospheric electromagnetic background noise in the range of short-period geomagnetic pulsations. Radiophys Quantum Electron 32:802-810

Belyaev PP, Polyakov SV, Rapoport VO, Trakhtengertz VY (1990) The ionospheric Alfvén resonator. J Atmos Terr Phys 52:781-787

Belyaev PP, Bösinger T, Isaev SV, Kangas J (1999) First evidence at high latitudes for the ionospheric Alfvén resonator. J Geophys Res 104:4305-4317

Belyaev PP, Polyakov SV, Ermakova EN, Isaev SV (2000) Solar cycle variations in the ionospheric Alfvén resonator 1985-1995. J Atmos Solar Terr Phys 62:239-248

Bilitza D, Reinisch B (2008) International reference ionosphere 2007: improvements and new parameters. Adv Space Res 42:599-609

Bösinger T, Demekhov AG, Trakhtengertz VY (2004) Ionospheric Alfvén resonator spectra observed at low latitude (L=1.3). Geophys Res Lett 31:L18802. doi:10.1029/2004GL020777

Bösinger T, Ermakova EN, Haldoupis C, Kotik D (2009) Inclination effects in the spectral resonance structure of the ionospheric Alfvén resonator. Ann Geophys 27:1313-1320

Chaston CC, Bonnell JW, Carlson CW et al (2002) Electron acceleration in the ionospheric Alfvén resonator. J Geophys Res 107:1413. doi:10.1029/2002JA009272

Demekhov AG, Belyaev PP, Isaev SV et al (2000) Modeling the diurnal evolution of the resonance spectral structure of the atmospheric noise background in the Pc 1 frequency range. J Atmos Sol Terr Phys 62:257-265

Demekhov AG (2012) Coupling at the atmosphere-ionosphere-magnetosphere interface and resonant phenomena in the ULF range. Space Sci Rev 168:595-609

Deminov MG, Oraevsky VN, Ruzhin YY (2001) Ionosphere-magnetosphere effects of rockets launched toward high latitudes. Geomagn Aeron 41:738-745

Dudkin D, Pilipenko V, Korepanov V, Klimov S, Holzworth R (2014) Electric field signatures of the IAR and Schumann resonance in the upper ionosphere detected by Chibis-M microsatellite. J Atmos Solar Terr Phys 117:81-87

Ergun RE, Su YI, Andersson L et al (2006) S bursts and the jupiter ionospheric Alfvén resonator. J Geophys Res 111:A06212

Ermakova EN, Kotik DS, Polyakov SV, Shchennikov AV (2007) On a mechanism forming a broadband maximum in the spectrum of background noise at frequencies 2-6 Hz. Radiophys Quantum Electron 50:555-569

Ermakova EN, Kotik DS, Polyakov SV (2008) Studying specific features of the resonance structure of the background noise spectrum in the frequency range 1-10 Hz with allowance for the slope of the earth's magnetic field. Radiophys Quantum Electron 51:519-527

Ermakova EN, Belyaev PP, Belova NI, Trakhtenherts VYu (2000) A sunset effect in variations of the eigefrequencies of the ionospheric Alfvén resonator. J Atmos Solar Terr Phys 62:277-298

Fedorov E, Pilipenko V, Engebretson MJ (2001) ULF wave damping in the auroral acceleration region. J Geophys Res 106:6203-6212

Fedorov EN, Schekotov AJ, Molchanov OA, Hayakawa M, Surkov VV, Gladyshev VA (2006) An energy source for the mid-middle latitudes IAR: world thunderstorm centers, nearby discharges or neutral wind fluctuations? Phys Chem Earth 31:462-468

Fedorov E, Schekotov A, Hobara Y, Nakamura R, Yagova N, Hayakawa M (2014) The origin of spectral resonance structures of the ionospheric Alfvén resonator: single high-altitude reflection or resonant cavity excitation? J Geophys Res 119:3117-3129. doi:10.1002/2013JA019428

Gaidukov VM, Deminov MG, Dumin YuV et al (1993) “Auroral trigger” experiment, 1. Generation of electric fields and particle fluxes by plasma injection into the ionosphere at high latitudes. Space Res Kosmicheskie Issledovaniya 31:54-62

Ginzburg VL (1970) The propagation of electromagnetic waves in plasmas. Pergamon Press, Oxford

Greifinger C, Greifinger PS (1968) Theory of hydromagnetic propagation in the ionosphere wave guide. J Geophys Res 73:7473-7490 
Heacock RR (1967) Two subtypes of type Pi micropulsations. J Geophys Res 72:3905-3917

Hebden SR, Robinson TR, Wright DM et al (2005) A quantitative analysis of the diurnal evolution of ionospheric Alfvén resonator magnetic resonance features and calculation of changing IAR parameters. Ann Geophys 23:1711-1721

Kulak A, Maslanka K, Michalec A, Zieba S (1999) Observations of Alfvén ionospheric resonances on the earth's surface. Stud Geophys Geod 43:399-406

Leroy B (1980) Propagations of waves in an atmosphere in the presence of a magnetic field. Astron Astrophys 91:136-146

Lessard MR, Knudsen DJ (2001) Ionospheric reflection of small-scale Alfvén waves. Geophys Res Lett 28:3573-3576

Lysak RL (1991) Feedback instability of the ionospheric resonator cavity. J Geophys Res 96:1553-1568

Lysak RL (1993) Generalized model of the Ionospheric Alfvén Resonator. In: Lysak RL (ed) "Auroral Plasma Dynamics", Geophysical Monograph 80. AGU, Washington, pp 121-128

Lysak RL, Waters CL, Sciffer MD (2013) Modeling IAR in dipole. J Geophys Res 118:1514-1528

Lysak RL, Yoshikawa A (2013) Resonant cavities and waveguides in the ionosphere and atmosphere. In: Takahashi K, Chi PJ, Denton RE, Lysak RL (eds) Magnetospheric ULF waves: synthesis and new directions. American Geophysical Union, Washington

Molchanov OA, Schekotov AYu, Fedorov EN, Hayakawa M (2004) Ionospheric Alfvén resonance at middle latitudes: results of observation at Kamchatka. Phys Chem Earth 29:649-655

Parent A, Mann IR, Rae IJ (2010) Effects of substorm dynamics on magnetic signatures of the ionospheric Alfvén resonator. J Geophys Res 115:A02312. doi:10.1029/2009JA014673

Pashin AB, Mochalov AA, Bösinger T, Rietveld MT (2003) Excitation of ionospheric Alfvén resonator by artificial magnetic pulsations, physics of auroral phenomena. In: Proceedings on XXVI annual seminar, Apatity, pp 111-114

Pilipenko VA (2012) Impulsive coupling between the atmosphere and ionosphere/magnetosphere. Space Sci Rev 168:533-550

Polyakov SV, Rapoport VO (1981) The ionospheric Alfvén resonator. Geomagn Aeron 21:816-822

Pokhotelov OA, Khruschev V, Parrot M, Senchenkov S, Pavlenko VP (2001) Ionospheric Alfvén resonator revisited: feedback instability. J Geophys Res 106:2156-2202

Potapov AS, Polyushkina TN, Dovbnya BV, Tsegmed B, Rakhmatulin RA (2014) Emissions of ionospheric Alfvén resonator and ionospheric conditions. J Atmos Solar Terr Phys 119:91-101

Schekotov A, Pilipenko V, Shiokawa K, Fedorov E (2011) ULF impulsive magnetic response at midlatitudes to lightning activity. Earth Planets Space 63:119-128

Sciffer MD, Waters CL, Menk FW (2005) A numerical model to investigate the polarisation azimuth of ULF waves through an ionosphere with oblique magnetic fields. Ann Geophys 23:3457-3471

Shiokawa K, Nomura R, Sakaguchi K et al (2010) The STEL induction magnetometer network to observe high-frequency geomagnetic pulsations. Earth Planets Space 62:517-524

Semenova NV, Yahnin AG (2008) Diurnal behavior of the ionospheric Alfvén resonator signatures as observed at high latitude observatory Barentsburg ( $L=15)$. Ann Geophys 26:2245-2251

Simões F, Klenzing J, Ivanov S et al (2012) Detection of ionospheric Alfvén resonator signatures in the equatorial ionosphere. J Geophys Res 117:A11305. doi:10.1029/2012JA017709

Surkov VV, Pochotelov OA, Parrot M et al (2004) Excitation of the ionospheric resonance cavity by neutral winds at middle latitudes. Ann Geophys 22:2877-2889

Surkov VV, Hayakawa M, Schekotov AJ et al (2006) Ionospheric Alfvén resonator excitation due to nearby thunderstorms. J Geophys Res 111:A01303. doi:10.1029/2005JA011320

Trakhtengertz VY, Feldstein A Ya (1991) Turbulent Alfvén boundary layer in the polar ionosphere, 1, excitation conditions and energetics. J Geophys Res 96:19363-19372

Trakhtengerts VY, Belyaev PP, Polyakov SV, Demekhov AG, Bösinger T (2000) Excitation of Alfvén waves and vortices in the ionospheric Alfvén resonator by modulated powerful radio waves. J Atmos Solar Terr Phys 62:267-276

Woodroffe JR, Lysak RL (2012) Ultra-low frequency wave coupling in the ionospheric Alfvén resonator: characteristics and implications for the interpretation of ground magnetic fields. J Geophys Res 117:A03223. doi:10.1029/2011JA017057

Yahnin AG, Semenova NV, Ostapenko AA et al (2003) Morphology of the spectral resonance structure of the electromagnetic background noise in the range of $0.1-4 \mathrm{~Hz}$ at $\mathrm{L}=5.2$. Ann Geophys 21:779-786 